\title{
Birgit Schultz \\ Ökonomische Voraussetzungen bäuerlicher Interessenvertretung in der BRD (1. Teil)
}

\section{Einleitung}

In der westdeutschen marxistischen Literatur, die die Probleme agrarischer Produktion erst in den letzten Jahren der Bedeutungslosigkeit entreißt, findet der Deutsche Bauernverband (DBV) als einzige politische Organisation der westdeutschen Bauern erstaunlich geringe Aufmerksamkeit. Die Agrarexperten der DKP, Richard Scheringer und Wilhelm Sprenger, vertreten die Auffassung, aus dem DBV eine „ebensolche Kampforganisation gegen das Monopolkapital" machen zu können, „wie das die fortschrittlichen Gewerkschaften für die Arbeiterklasse sind“(1), wenn man nur den Einfluß der „CDU/CSU-hörigen adligen Großgrundbesitzer", die die bauernfeindliche Politik des Verbandes bestimmen, zurückdämme (2/3). Daraus spricht zum einen die für die Theorie des Staatsmonopolistischen Kapitalismus typische „Subjektivierung des Kapitalbegriffs", d.h. dem realen Schein aufzusitzen, die gesellschaftlichen Verhältnisse seien bestimmt von einer kleinen Gruppe von Kapitalmagnaten und nicht als Totalität durch das Kapital als selbstverwertendem Wert (4), und zum andern bringt diese Einschätzung den theoretischen und praktischen Eklektizismus von den wissenschaftlichen Vorarbeiten aus der DDR (5) zum

1 Richard Scheringer/Wilhelm Sprenger, Arbeiter und Bauern gegen Bosse und Banken. Frankfurt 1970, S. 163

2 ibid., S. 128

3 Auf der 8. Tagung des Parteivorstandes der Deutschen Kommunistischen Partei am 27./28. Febr. 1971 in Düsseldorf wurde das ,Demokratische Bauernprogramm der DKP“ verabschiedet, das der „Bauernschaft als wichtigstem Verbündeten der Arbeiterklasse * (S. 5) gilt. - Seit Anfang 1971 gibt der Parteivorstand die „DKP-Landrevue - Informationen für die Landbevölkerung" heraus.

4 Vgl. Margaret Wirth, Zur Kritik der Theorie des staatsmonopolistischen Kapitalismus. In: Probleme des Klassenkampfs, Nr. 8/9, Berlin 1973, S. 26 (Zitat) und 28.

Als Beispiel für die "Subjektivierung des Kapitalbegriffs" aus dem thematischen Zusammenhang: „Die CDU/CSU-hörige Bauernverbandsführung, die Großgrundbesitzer im Bauernverband, haben sich mit dem BDI gemeinsam dafür ausgesprochen, die Bauernschaft den wachsenden Profit- und Machtwïnschen des herrschenden Großkapitals auch in Zukunft weiter zu unterwerfen". W. Sprenger, Das Bündnis von Arbeiterklasse und Bauernschaft im staatsmonopolistischen Kapitalismus. In: Marxistische Blätter, Nr. 5, Frankfurt 1972, S. $30 \mathrm{f}$.

5 Die DWI-Berichte aus dem Institut für Internationale Politik und Wirtschaft (jetzt IPWBerichte) enthalten seit Jahren empirische Untersuchungen und theoretische Einschätzungen des landwirtschaftlichen Sektors und der Agrarpolitik der BRD. Hinzuweisen sind vor allem auf die umfassenden Arbeiten Emil Rechtzieglers: „Neue Entwicklungstendenzen in der Landwirtschaft der kapitalistischen Staaten". Berlin 1968 und ders., Westdeutsche Landwirtschaft im Spätkapitalismus. In: DWI-Forschungsheft, Nr. 4, 1969 (im Folgenden zitiert nach Abdruck des Aufsatzes durch den Plakat-Bauernverlag, Heft 2.) 
Ausdruck, die einen in diesem Zusammenhang nicht näher zu bestimmenden Einfluß der sog. Politik des Bündnisses aller werktätigen Klassen und Schichten beim Aufbau des Sozialismus in der DDR aufweisen (6). Mehr als kritischer Reflex auf die Theorie des Staatsmonopolistischen Kapitalismus und die antimonopolistische Bündnispolitik der DKP denn aus Anlaß der massiven und zahlreichen Bauerndemonstrationen 70/71 in der BRD findet die ökonomische Situation der westdeutschen Bauern spät Eingang in Theorie und Praxis weiterer Kreise der bundesrepublikanischen Linken (7), eine Analyse der „Gewerkschaft“" (8) der Bauern steht - sieht man von den folgenden sporadischen Bemerkungen $a b$ - noch aus. In vermeintlicher Stoßrichtung gegen die DKP wird der DBV von der ,,antirevisionistischen" Linken als "Interessenvertretung der kapitalistischen Bauernbetriebe" (9) und als „Brückenkopf der in der Landwirtschaft engagierten Kapitalfraktion“"(10) entpuppt, - eine Inkonsistenz der Beurteilung, die bei Ulf Baumgärtner an anderer Stelle darin kulminiert, den DBV als „Klassenorgan der Großbauern und damit der Bourgeoisie“ (11) zugleich zu charakterisieren mit der Begründung, die DBVFührung arbeite in Interessengemeinschaft mit dem BDI (12). Diese Aussage unterscheidet sich von der Rechtsziegler'schen, der Bauernverband diene der CDU/CSU nur dazu, ,die agrarpolitische Konzeption des Monopolkapitals durchzusetzen“ (13), nur dadurch, daß hier gleich das gesamte Monopolkapital die DBV-Politik bestimmt, während es bei Baumgärtner nur eine Fraktion des Kapitals ist, wobei gerade das Problem der eklatanten Interessenkollisionen von Agrarkapitalisten und den übrigen Kapitalfraktionen auf der Ebene des Marktes unterschlagen wird.

6 Die theoretische Befassung mit dem Agrarsektor und der Eingang der Bauern in kommunistische, sozialistische und sozialdemokratische Parteiprogramme steht offenbar in Korrelation mit einem entwickelten Stand der Klassenkämpfe, der die Machtfrage akut macht. Vgl. die Diskussion der Agrarfrage in den 20er Jahren, die noch aufzuarbeiten ist. - Zurn Problem der Agrarfrage in der Arbeiterbewegung siehe als Uberblick Teil 2 und 3 der Dissertation von Max Kemper, Marxismus und Landwirtschaft. Bonn 1929 (veröffentlicht im Plakat-Bauernverlag, Heft 4, 1973).

7 Im Wesentlichen handelt es sich um die Veröffentlichungen:

- Monika Baumgartner, Entwicklungstendenzen in der westdeutschen Landwirtschaft. In: Probleme des Klassenkampfs, Nr. 3, Berlin 1972

- Onno-Hans Poppinga, Zur Bauernfrage in Westdeutschland. In: Bauer was nun? Beiträge zur Agrarfrage in der BRD. Plakat-Bauernverlag, Heft 3, Stuttgart 1972

- Ulf Baumgärtner, Vorwort zu: Emil Rechtziegler, Westdt. Landwirtschaft . . , a.a.O., o.S.

- ders., Anmerkungen zur Bauernpolitik der DKP. In: Probleme des Klassenkampfs. Nr. 8/9, Berlin 1973

8 Bezeichnung für den DBV in persönlichen Gesprächen der Verfasserin mit Landwirten.

9 O.-H. Poppinga, a.a.O., S. 27

10 U. Baumgärtner, Vorwort zu ..., a.a.O., o.S.

11 ders., Anmerkungen ... a.a.O., S. 195

12 ibid., S. 196

13 E. Rechtziegler, Westdt. Landwirtschaft ..., a.a.O., S. 104 
Angesichts des ungeklärten Widerspruches zwischen Attacken gegen den DBV als Sachwalter staatlicher Sonderbehandlung der Landwirtschaft, die gerade von der dem westdeutschen Kapital verpflichteten Presse vorgebracht werden (14), und der organisatorisch fixierten Zusammenarbeit mit Industrieverbänden und Kapitalfraktionen und im Hinblick auf die Ergebnisse einer Umfrage im Winter 69/70, in der $85 \%$ der befragten Bauern der Meinung war, der Deutsche Bauernverband setze sich wirkungsvoller für ihre Interessen ein als eine der politischen Parteien (15), erweist sich die Fragwürdigkeit einer auf soziologischen Untersuchungen der Elite beschränkten Einschätzung einer politischen Organisation, die sich in nichts von Analysen bürgerlicher Politologie unterscheidet. Daß die ausführlichen ökonomischen Analysen, in deren Kontext die oben genannten Aussagen über den DBV erscheinen, für letztere derart folgenlos bleiben, dürfte auch in der methodischen Schwäche dieser Arbeiten begründet liegen, der logischen Differenz zwischen den konkreten Erscheinungsformen agrarpolitischer Staatstätigkeit, der dem realen Schein der Oberfläche der bürgerlichen Gesellschaft verhaffteten Interessenartikulation und den allgemeinen Bewegungsgesetzen des Kapitals nicht Rechnung getragen zu haben, was sich dann in einer Vereinfachung wie: „Die westdeutsche Monopolbourgeosie geht ganz allgemein dazu über, die Existenzbedingungen der Bauern zu verschlechtern, um sie wirtschaftlich zu zwingen, sich ihrer agrarpolitischen Konzeption zu unterwerfen" (16) niederschlägt.

Ausgehend von der Kritik an dieser deskriptiv-politizistischen Metbode zur Einschätzung einer politischen Organisation, (die nicht ein ähnliches Ergebnis ausschließt), soll das Ziel dieses Aufsatzes sein, die Tätigkeit des DBV als Aggregierung von Interessen abzuleiten, die herrühren aus der objektiven Schranke, die die in der BRD vorherrschende Form agrarischer Produktion als einfacher Warenproduktion für die Ausdehnung der kapitalistischen Produktionsweise darstellt. Die maßlose Tendenz des Kapitals, sich alle Sphären der Produktion zu unterwerfen, zeitigt notwendig Interessenkollisionen zwischen den Eigentümern der Revenuequellen, sei es der Besitzer von Kapital untereinander auf der Ebene der Konkurrenz der Einzelkapitale einer Sphäre, sei es ihrer gemeinsamen Interessen an möglichst großer

14 Die FAZ argumentiert durchgängig gegen die Ansprüche der Landwirtschaft und ihrer Interessenvertretung. Siehe dazu als Bsp. den Artikel von: Klaus Peter Krause, Heeremann auf sanfter Welle. In: FAZ vom 9.7.73 - Vgl. auch Artikel im "Spiegel"

Die einzigen Gesamtdarstellungen über den DBV, die allerdings aus der Zeit besonderer Interessendivergenzen stammen, stellen übereinstimmend ein gespanntes Verhältnis des DBV zum DBI und DIHT heraus:

- Peter Christof Lutz, Der Deutsche Bauernverband. Diss. Mannheim 1967, S. 188

- Hans Bürger, Die Landwirtschaftliche Interessenvertretung in der Zeit von 1933 bis zur Gegenwart unter besonderer Berücksichtigung der westdt. Verhältnisse. Erlangen/Nürnberg 1967, S. 242

- Paul Ackermann, Der Deutsche Bauernverband im politischen Kräftespiel der BRD. Tübingen 1970, S. 65 (Die Arbeit wurde lt. Angabe des Verf. im Herbst 67 abgeschlossen, Seite V).

15 "Bericht zur Untersuchung über die Einstellung von Landwirten zu agrarpolitischen Fragen“. Bonn Febr. 1970, S. 19 (unveröffentl. Manuskript), zitiert bei H.-O. Poppinga, Zur Bauernfrage . . , a.a.O., S. 29

E. Rechtziegler, Westdt. Landw. . ., a.a.O., S.,97 
Mehrwertabpressung und Mehrwertrealisierung gegenüber den Kapitaleigentümern anderer Produktionssphären. Diesen Gruppen, deren Privateigentum auf der Aneignung fremder Arbeit beruht, stehen (nicht antagonistisch im Sinne der Lohnarbeit) gegenüber die Gruppe der einfachen Warenproduzenten, die zwar Besitzer von Produktionsmitteln sind, deren Eigentum aber Resultat eigner Arbeit ist. Auf der Oberfläche der bürgerlichen Gesellschaft, der Ebene der Zirkulation, auf der in der „fetischartigsten Form" (17) die wirkliche Quelle des jährlich geschaffenen Neuwerts verschwindet, und dieser als Resultat der drei Revenuequellen Arbeit, Boden und Kapital erscheint, konstituiert sich die Sphäre der Interessenartikulation der Privateigentümer, die ausgerichtet ist auf die optimale Nutzung ihrer Revenuequellen. Auf dieser Ebene der Gesellschaftlichkeit, die in der Kategorie des Interesses Resultat der logischen Differenz ist zwischen Charaktermasken als Personifikationen allgemeiner ökonomischer Gesetzmäßigkeiten und Subjekten, die in einem gewissen Spielraum über die optimale Nutzung ihrer Revenuequelle bestimmen können, ist die Möglicbkeit der Interessenvertretung eines Verbandes wie des DBV anzusiedeln. Die inhaltliche Bestimmung seiner Forderungen ist methodisch richtig abzuleiten von den Erfordernissen des Produktions- und Reproduktionsprozesses des Kapitals auf einer bestimmten Stufe seiner Entwicklung.

Nachdem ich zunächst die logische Differenz zwischen der Ebene der allgemeinen Bewegungsgesetze des Kapitals und der Oberfläche der bürgerlichen Gesellschaft, auf der sich in den Aktionen der „freien Subjekte“ die Erfordernisse der Kapitalverwertung in der für die kapitalistische Gesellschaft typischen Form des Verschwindens der Eigentums- und Aneignungsverhältnisse im Produktionsprozeß als Voraussetzung dieses Aufsatzes abgeklärt habe, will ich die daraus resultierenden methodischen Implikationen am Gang der Analyse erläutern.

Der erste Teil des Aufsatzes, 2. „,die ökonomische Lage der Landwirtschaft in der BRD", befaßt sich mit den historisch besonderen Erscheinungsformen der immanenten Bewegungsgesetze des Kapitals, wie sie sich beim Eindringen der kapitalistischen Produktionsweise in die ,,aus der vorkapitalistischen in die bürgerliche Gesellschaft geretteten Bereiche einfacher Warenproduktion und reiner Gebrauchswerterzeugung" (18) durchsetzen. Von dieser Ebene aus ist es analytisch möglich, die Kategorien von Produktionsagenten zu entwickeln, die in ihrer Eigenschaft als Revenuequellenbesitzer ihre je besonderen Interessen, die sich aus den Anforderungen an Sicherung und Nutzung ihrer Revenuequelle ergeben, artikulieren und aufgrund der Privatheit der kapitalistischen Produktion in Konkurrenz treten. Diese Konkurrenz ist nur in einigen Fällen gleichzusetzen mit der Konkurrenz der Einzelkapitale als Form der Durchsetzung des Wertgesetzes, vielmehr handelt es sich um die (Distributions-) Konkurrenz der drei Kategorien von

17 Karl Marx, Theorien über den Mehrwert. In: Marx-Engels-Werke (MEW), Bd. 26.3, Berlin 1969, S. 445. (Im folgenden werden Zitate nur noch mit MEW . . . . angegeben).

18 Ernest Mandel, Der Spätkapitalismus. Frankfurt 1972, S. 344. Das Stadium reiner Gebrauchswerterzeugung, der Subsistenzwirtschaft, muß in der BRD als abgeschlossen gelten. Vgl. dazu M. Baumgartner, a.a.O., S. $57 \mathrm{f}$ 
Revenuequellenbesitzern untereinander um den größtmöglichen Anteil am geschaffenen Neuwert (Ebene der Herausbildung von Klassen) oder um die der Koalition von Besitzern verschiedener Revenuequellen einer Sphäre mit der einer andern um Verbesserung der Konkurrenzvoraussetzungen (auf der Ebene der allg. Bewegungsgesetze des Kapitals Ausgleich der Profitraten) (19). Auch andere Kombinationen sind denkbar. Mit der Analyse der konkreten Erscheinungsformen ist die Basis der Bestimmungen der möglichen Interesseninhalte der agrarischen und nicht-agrarischen Produktionsagenten entwickelt, die sich zu allgemeinen Interessen bestimmter Gruppen zusammenfassen lassen, die sich der DBV dann unter Ausschluß konkurrierender Interessen zu eigen machen und als spezifisches Gruppeninteresse artikulieren kann.

Die Durchsetzung allgemeiner Interessen kann aufgrund der Privatheit der Produktion in der kapitalistischen Gesellschaft nur über die Institution des Staates als Zusammenfassung der bürgerlichen Gesellschaft, die als besondere Form abgehoben von dieser existiert, erfolgen, dem die Verwaltung und Verfolgung der allgemeinen Interessen obliegt (Teil 3).

Auch wenn im Einzelnen nicht immer ausgeführt, geht in die Analyse der Staatstätigkeit immer die Voraussetzung ein, die aus der Formbestimmtheit des Staates durch die kapitalistische Produktionsweise abgeleitet ist, daß nur solche Interessen tendenziell (restringiert durch den Zwang der Vermeidung sozialer Kämpfe zur Erhaltung der kapitalistischen Produktionsweise als Ganzer) durchgesetzt werden können, die auf die Aufhebung von Schranken in Zirkulation und Produktion für den Verwertungsprozeß des Gesamtkapitals zielen, eine Bestimmung, die Größe, Organisationsgrad oder ,Schlagkraft“ eines Interessenverbandes in ihrer Bedeutung für seine Funktionsfähigkeit herabsetzt und diese soziologischen Kategorien allein als analytisch untaugliche Instrumente erweist.

Teil 4. der Analyse stellt zunächst einen Bruch im allgemeinen Ableitungszusammenhang dar, der der Unmöglichkeit geschuldet ist, politische Phänomene, wie sie Interessenverbände sind, in ihrer real-historischen Verfaßtheit logisch aus den ,allgemeinen Bestimmungen des Kapitals, realen Durchsetzungsformen (Konkurrenz, Monopol, d.Verf.) und Erscheinungsweise dieser Durchsetzung" (20) abzuleiten. Auf der Stufe der Analyse des DBV selbst erhält die phänomenologischempirische Methode ihre partielle Berechtigung zur Deskription seiner Organisationsstruktur, seinen inhaltlichen Forderungen und ihrer Begründung und der diversen Strategien zur Interessendurchsetzung.

Die Beziehung der konkret-historischen Erscheinungsformen der Interessenvertretung durch den DBV auf die Schwierigkeiten, die sich für die jeweiligen Kategorien von Revenuequellenbesitzern aus der Durchsetzung der kapitalistischen Produktionsweise in der Landwirtschaft ergeben (Teil 2.), soll im Schlußteil erfolgen, um die objektive Funktion des DBV in diesem Prozeß aufzuhellen. Auch

19 Diese setzen sich aufgrund ökonomischer und außerökonomischer Schranken immer nur der Tendenz nach durch.

20 Margaret Wirth, Zur Kritik der Theorie . . a.a.O., S. 20 
wenn die real-erscheinenden Phänomene im Prozeß der Erkenntnis immer nur a posteriori auf die objektiven Anforderungen der Kapitalverwertung bezogen werden können, haben wir uns mit der analytischen Trennung von Bewegungsformen des Kapitals und dem Agieren der Subjekte als Privateigentïmer, die dem realen Schein der Gleichheit und Freiheit auf der Oberfläche der bürgerlichen Gesellschaft, die die reale Aneignungsweise verkehrt, verfallen, in die Lage versetzt, die Illusionen zu erklären, die sich an die Möglichkeit „antikapitalistischer Politik“ eines Interessenverbandes wie des DBV knüpfen (21).

\section{Die ökonomische Lage der Landwirtschaft in der BRD}

\subsection{Die natürliche Sonderstellung agrarischer Produktion}

Die agrarische Produktion unterscheidet sich gegenüber allen Sphären industrieller Produktion durch besondere natürliche Bedingungen, die die Durchsetzung der kapitalistischen Produktionsweise verzögern und modifizieren, aber als Warenproduktion sind „Landwirtschaft und Industrie gemeinsame Glieder eines Gesamtprozesses" (22). Die vermeintliche Eigengesetzlichkeit der Agrikultur, die erscheint ,als Naturprozeß, den der Mensch leitet, obgleich er ihn auch, ein bißchen' macht"“ (23), schlug sich schon in der Agrardebatte der Sozialdemokratie vor dem I. Weltkrieg um die Überlegenheit von Groß- oder Kleinbetrieb, an der sich die Geltung der von Marx formulierten Entwicklungsgesetze des Kapitals erweisen sollte, nieder (24), und wird in der modernen bürgerlichen Agrarökonomie zum Apriori der sog. „Pessimisten“ (25), die aus dem „Abstand zwischen Landwirtschaft und Industrie

21 Damit meine ich sowohl die Hoffnung der DKP, den DBV zu einem anti-monopolistischen Kampfverband umfunktionieren zu können (vgl. Scheringer/Sprenger a.a.O., S. 128), als auch Positionen im DBV selbst, die in einer ideologischen Tradition stehen, wie sie Rehwinkel, ehemaliger Präsident des DBV, formulierte: „. . . denn die Eigentumsgebundenheit des bäuerlichen Wirkens und Denkens hat das Bauerntum zum festesten Bollwerk einer Gesellschaftsordnung gemacht, die im Eigentumsgedanken wurzelt... Dieser starke Unterbau kleiner und kleinster Eigentümer ist das feste Fundament, auf dem das größere Eigentum steht, und wenn immer wieder von Stimmen, die vermeintlich das Interesse der Industrie im Auge haben, einem Agrarstrukturwandel das Wort geredet wird, ..., so sollten sich die führenden industriellen Persönlichkeiten bewußt sein, daß damit die Basis zerstört würde, auf der sie stehen". Vgl. Edmund Rehwinkel, Gegenwartsfragen der Deutschen Agrarpolitik. In: Festschrift für Andreas Hermes. Neuwied 1959, S. $378 \mathrm{f}$. -

22 Karl Kautsky, Die Agrarfrage. Stuttgart 1899 (photomechanischer Nachdruck, Graz 1971), S. 6 - und Max Kemper, a.a.O., S. 14

23 MEW 26.2., S. 57

24 Vgl. dazu Karl Kautsky, a.a.O. S. 4 ff

25 Die Unterscheidung von Optimisten und Pessimisten in der Beurteilung landwirtschaftlicher Entwicklungsmöglichkeiten gehört zu den Allgemeinplätzen bürgerlicher Agrarökonomie. Siehe z.B.: Karl-Heinz Hansmeyer, Finanzpolitische Aspekte der Agrarförderung. In: Agrarwirtschaft und Agrarpolitik hrsg. von E. Gerhardt/P. Kuhlmann. Köln/Berlin 1969, S. 224 f und P. Ch. Lutz, a.a.O., S. 30 f. 
als ,naturgegeben" und demnach unabänderlich“ (26) den Zwang zu permanenter staatlicher Unterstützung herleiten, während die sogenannten „Optimisten die Ursachen landwirtschaftlicher Disparität vorwiegend in der grundsätzlich verbesserungsfähigen agrarischen Betriebsstruktur, die durch mangelnde Rationalität gekennzeichnet sei" (27), sehen und das Heil in einer entsprechenden Anpassungspolitik suchen.

Der Charakter organiscber Natur, der die Produkte, Produktionsmittel und technologischen Bedingungen agrarischer Produktion auszeichnet, stellt ein spezifisches Hindernis dar für die industrielle Produktion und erst recht für deren kapitalistisch betriebene Form (28). Dieses Hindernis ist von gesellschaftlichen und kapitalimmanenten Schranken nur analytisch abtrennbar, da die gesellschaftliche Form der Produktion, wie z.B. der Familienbetrieb, gerade dann als Folge dieser Spezifika anzusehen ist, wenn es sich als historisch so schwer überwindbar erweist. Angesichts der Nicht-Systematisierbarkeit im Sinne einer besonderen Agrartheorie seien im Folgenden die wichtigsten Faktoren und ihre Auswirkungen auf den Prozeß der Vergesellschaftung der Produktion genannt (29).

a) Das Eigentum am Boden unterscheidet sich von jedem andern Privateigentum dadurch, daß der Boden nicht beliebig vermehrbar ist. Damit ist die Möglicbkeit zu seiner Monopolisierung in Form des Grundeigentums freigesetzt, die eine Schranke für den Ausgleich der Profitraten bildet, d.h. Surplusprofit ermöglicht. Voraussetzung der absoluten Rente ist, daß der Boden der Bebauung so lange entzogen wird, bis der Marktpreis der Produkte über ihren Wert steigt oder auch nur über ihren Produktionspreis und die organische Zusammensetzung des Kapitals in der Sphäre der Agrikultur niedriger ist als in anderen Bereichen (30). Bei der kleinbäuerlichen Wirtschaftsweise heute existiert die absolute Rente vermutlich nicht (31), da die bäuerlichen Produzenten ihren Boden auch dann noch bebauen, wenn er ihnen nur die Reproduktionskosten des Betriebs und ihrer Arbeitskraft abwirft (32).

b) Der Jahreszyklus der Feld- und der Mehrjahreszyklus der Viehproduktion bedingen eine Differenz von Arbeits- und Produktionszeit, die, da nur die aufgewandte Arbeitszeit die Wertgröße des Produkts bestimmt, (inclusive der Arbeits-

26 K.-H. Hansmeyer, Finanzpolitische . . a.a.O., S. 22

27 P. Ch. Lutz, a.a.O., S. 31

28 „Der ganze Geist der kapitalistischen Produktion, der auf den unmittelbaren nächsten Geldgewinn gerichtet ist, widerspricht der Agrikultur, die mit den gesamten ständigen Lebensbedingungen der sich verkettenden Menschengenerationen zu wirtschaften hat ${ }^{\text {". }}$ MEW 25, S. 631, Fußnote 27.

29 Empirische Basis sind die durchschnittlichen Produktionsbedingungen in der BRD/EWG 1975, nicht die fortgeschrittene industrielle Produktion von Geflügel etc. und die in der Forschung anvisierten sogenannten „Agrarfabriken“.

30 Vgl. MEW 25, S. 756-780

31 Die Pacht ist zwar nominell Grundrente, die Existenz einer Rente ist für die einzelnen Produzenten aber nicht Bedingung für die Obernahme von Pachtland; dessen Kosten bestreitet er im Extremfall aus dem Abzug von seinen Reproduktionskosten.

Vgl. E. Mandel, Der Spätkapitalismus, S. 349 a.a.O. 
zeit, die in den Produktionsmitteln steckt) sich in relativ niedriger Produktivität pro Produktionsphase niederschlägt und zugleich die Zunahme der Umschlaggeschwindigkeit des Kapitals behindert, so daß die „Agrikultur nie die Sphäre sein kann, mit der das Kapital beginnt ... Es widerspricht dies den ersten Grundbedingungen der industriellen Arbeit", steht ,also direkt der Verwertung der Arbeit im Weg. .., d.h. der Aneignung von Surplusarbeit durch das Kapital" (33). Die lange Produktionszeit bedingt zum andern, in Verbindung mit der zersplitterten Produktion, eine relative Inelastizität des Angebots und damit ein erhebliches Absatzrisiko aufgrund der Preisschwankungen, ein Phänomen, das unter dem Begriff des „Schweinezyklus" einen Großteil der agrarischen Produktion betrifft.

c) Auf der Konsumentenseite muß erwähnt werden die unter kapitalistischen Bedingungen relativ stagnierende Nachfrage nach agrarischen Grundernahrungsgütern (formuliert im sogenannten Engelschen Gesetz: die Einkommenselastizität für Nahrungsmittel ist kleiner als 1), die infolge wachsender Produktivität der Arbeit bei gleichbleibender zahlungsfähiger (!) Nachfrage agrarische Arbeitsk räfte freisetzt.

d) Im Produktionsprozeß wird die Teilung der Arbeit und die Anwendung von Maschinerie als Voraussetzung industrieller Arbeit behindert durch die jahreszeitlich bedingte Nichtauslastung der Maschinenkapazitäten (34) und den Reifeprozeß organischer Produkte, der eine Spezialisierung im „Innern der Gesellschaft" und eine Zerlegung der Arbeit im „Innern des Ateliers“" (35) nur bis zu einer bestimmten Grenze zuläßt. Angesichts der Entwicklung der Chemie, Geologie und Physiologie zu Ende des 19. Jh. als wissenschaftliche Grundlagen der Agrikultur nimmt Marx eine Beschleunigung der landwirtschaftlichen Produktivität gegenüber der Industrie an (36), die allerdings nur inbezug auf die Arbeitszeit gilt, die infolge des organischen Wachstums Phasen von Nicht-Arbeitszeit zur technologischen Bedingung hat (37). Neben der Monopolisierung des Produktionsmittels Boden ist es die Besonderheit der Natur, die Marx immer wieder als Ursache geltend macht, daß die kapitalistische Produktionsweise sich der Landwirtschaft erst bemächtigt, wenn ,sie alle Sphären der Produktion und der bürgerlichen Gesellschaft beherrscht" (38), wie es auch Friedrich Engels als Nachtrag zum 3. Bd. des „Kapital“" expressis verbis in der Erklärung formuliert, warum sich das Kapital nicht zunächst der theoretiscb profitabelsten Sphäre zuwendet: „,Denn die Produktionssphären mit

Karl Marx, Grundrisse der Kritik der politischen Ökonomie. Frankfurt o.J., S. 561 f. (im weiteren zitiert als: Grundrisse).

34 Das gilt in beschränktem Umfang, aber grundsätzlich auch für ihre Anwendung in Großbetrieben.

35 Karl Marx, Resultate des unmittelbaren Produktionsprozesses. Frankfurt 1969, S. 92

„Auf einem gewissen Höhepunkt der Industrie muß die Disproportion abnehmen, d.h die Produktivität der Agrikultur sich relativ rascher vermehren als in der Industrie.. Erst im 19. Jh., speziell in den späten Jahrzehnten, entwickeln sich die Wissenschaften, die direkt in höherem Grade spezifische Grundlagen für die Agrikultur als für die Industrie sind - Chemie, Geologie und Physiologie“. MEW 26.2., S. 103

38 MEW 25, S. 627 
überschüssigem Mehrwert, also mit hohem variablem bei niedrigem konstantem Kapital, also mit niedriger Kapitalzusammensetzung, sind gerade ibrer Natur nach diejenigen, die dem kapitalistischen Betrieb am spätesten und am unvollständigsten unterworfen werden; vor allem der Ackerbau“"(39).

Die Erarbeitung der grundlegenden natürlichen Besonderheiten des Agrarsektors wendet sich zum einen kritisch gegen die Richtung bürgerlicher Agrarökonomie, die alle Argumente der ,pessimistischen“ Entwicklungstheorie als widerlegbar betrachtet (40), will aber andererseits nicht Parteinahme sein für die zum Glaubenssatz erhobene Erklärung der Disparität durch den Deutschen Bauernverband: „... weil das Kernstück aller agrarischen Produktion - das Keimen und das Wachsen, das Bliihen und das Fruchtbringen - nicht vom Menschen gemacht werden kann. Die industrielle Produktion hingegen ist in ihrem vollen Ablauf Gegenstand menschlichen Tuns" (41). Vielmehr sollen unter Berücksichtigung der in der BRD vorfindlichen Agrarstruktur (42) spezifische Voraussetzungen für die Entwicklung im Agrarsektor abgeklärt werden.

2.2 Probleme vorkapitalistiscber Produktionsweisen unter den Bedingungen des Spätkapitalismus

Die Entwicklungsschwierigkeiten des bundesrepublikanischen Agrarsektors als

39 Friedrich Engels, Nachtrag zum Kapital Bd. 3. In: MEW 25, S. 916 (Hervorhebung durch den Verf.)

40 Vgl. K.-H. Hansmeyer, Finanzpolitische Aspekte . ., , a.a.O., S. 224

41 Deutsche Bauernkorrespondenz (dbk). Monatsschrift des Deutschen Bauernverbandes. Heft 12, 1965, S. 137. Zitiert bei: P. Ch. Lutz, a.a.O., S. 95 (Zitate aus der Deutschen Bauernkorrespondenz erscheinen im Folgenden nur gekennzeichnet mit dbk . . .) .

42 Auf die historische Entstehung der spezifisch bundesrepublikanischen Agrarstruktur kann im Rahmen dieses Aufsatzes nicht näher eingegangen werden.

Obwohl das historische Niveau des bäuerlichen Parzelleneigentums, das Marx im Grundrentenkapitel im Auge hat, überschritten ist und einige Beschränkungen dieser Wirtschaftsweise entfallen sind, gelten die grundlegenden Bestimmungen m.E. auch für die bäuerliche Agrarstruktur heute (insbesondere: Produktion auch bei Nichterwirtschaftung von Profit, Boden als Existenzgrundlage etc.). 
historisch-konkrete Erscheinungsformen sind zu entwickeln aus den logischen Bestimmungen der Disparität von vorwiegend nicht-kapitalistischer Produktionsweise und kapitalistischen Austauschverhältnissen (43) und ihrer Aufhebung durch tendenzielle Subsumtion von Produktion und Produktionsagenten unter die herrschende Produktionsweise. Kennzeichen der westdeutschen Agrarstruktur ist eine trotz Konzentrationstendenzen - nach wie vor starke Parzellierung der Agrarstruktur, wie eine Aufschlüsselung nach Größenklassen zeigt:

Übersicht 1: Zahl der landwirtschaftlichen Betriebe nach Größenklassen in ha landw. Nutzfläche (Zahl der Betriebe in 1000$)(44)$

\begin{tabular}{rrrrrr} 
& -5 ha & $5-10$ ha & $10-20$ ha & $20-30$ ha & $30-50$ \\
\cline { 2 - 6 } 1949 & 1151,5 & 403,8 & 256,2 & 72,1 & 40,3 \\
1972 & 598,9 & 205,9 & 243,1 & 108,5 & 61,3
\end{tabular}

1949

$$
50-100 \text { ha }
$$

100 ha und mehr

1972

$$
12,6
$$$$
3,0
$$

$$
18,9
$$

3,4

Unstreitig ist davon auszugehen, daß, ,in der Landwirtschaft im großen und ganzen noch Produktionsverhältnisse vor(-herrschen), deren Grundzüge der vorkapitalistischen Gesellschaftsordnung entstammen" (45), d.h. der Bauer ist unmittelbarer Produzent und Privateigentümer sowohl seiner Arbeitsmittel als auch des Bodens (46/47). Der Begriff der bäuerlichen ,einfachen Warenproduktion“ ist als kategoriale Bestimmung zu fassen, die zwar sektoral historische Realität besitzt und

43 Sobald "die Zirkulation als entwickeltes Moment" existiert, hat sie die Tendenz, ihre eigenen Voraussetzungen zu schaffen. Grundrisse, S. 631 und 167

44 Berechnet und zusammengestellt nach: Deutscher Bundestag, 7. Wahlperiode, Materialband zum Agrarbericht 1973 der Bundesregierung, Drucksache 7/147, Tab. 108, S. $224 \mathrm{f}$

45 E. Rechtziegler, Westdt. Landwirtschaft ..., a.a.O., S. 15. - Diese Meinung wird von allen marxistischen Autoren, die sich in jüngster Zeit mit der Landwirtschaft in der BRD en détail befaßt haben, geteilt. Vgl. z.B.:

- M. Baumgartner, a.a.O., S. $70 \mathrm{f}$

- U. Baumgärtner, Anmerkungen ... a.a.O., S. 166

- H.-O. Poppinga, Zur Bauernfrage ... a.a.0., S. 4

46 Vgl. die Marxschen Bestimmungen der einfachen Warenproduzenten $u_{.}$. in: MEW 23, S. 789 und MEW 25, S. 812 ff

47 Von verschiedenen Formen partieller Expropriation (Hypotheken, Zinsen für Leihkapital etc.) und Proletarisierung (Vertragslandwirtschaft, Nebenerwerb etc.) sei auf dieser Ebene der Analyse zunächst abgesehen, da sie die grundlegenden Bestimmungen nicht berïhren. 
besessen hat, aber keine Aussage über die Existenz einer ganzen historischen Phase der einfachen Warenzirkulation und -produktion als der kapitalistischen Produktionsweise vorhergehend impliziert (48). Der Auflösungsprozeß vorkapitalistischer Produktionsweisen muß als Reflex begriffen werden auf die ihr äußerliche kapitalistische Produktionsweise - und zwar zum einen vermittelt über die Ebene der Zirkulation (49), zum andern durch direkte Übernahme ehemals bäuerlicher Produktion als industrielle durch das Kapital.

Auf der Ebene der Zirkulation, auf der sich gemäß dem Wirken des Wertgesetzes der gesellschaftliche Zusammenhang der Produktion im Nachhinein herstellt, tritt der selbständige Landwirt als einfacher Warenproduzent auf, der seine Ware gegen ein Äquivalent in Form des Geldes (sei es der Herkunft nach Geld als Revenue oder Geldkapital) tauscht, um dieses wiederum auszutauschen gegen Waren als Produktionsmittel zur Reproduktion oder erweiterten Reproduktion seines Betriebes oder zum Zweck seiner eigenen Reproduktion. „Die Zirkulation, die also als das unmittelbar Vorhandene an der Oberfläche der bürgerlichen Gesellschaft erscheint, ist nur ... die Vermittlung vorausgesetzter Extreme. Aber sie setzt die Extreme nicht" $(50)$. Sie ermöglicht also in ihrer Funktion der Vermittlung von Waren - gleichgültig von wem die in ihnen vergegenständlichte Arbeit herrührt die Existenz verschiedener Eigentums- und Aneignungsverhältnisse innerhalb der Produktionsprozesse nebeneinander. Obgleich der selbständige Warenproduzent als Nicht-Kapitalist nicht am Durchschnittsprofit als notwendige Bedingung seiner Produktion orientiert ist (51), nehmen seine Waren auf der Basis der kapitalistischen Produktion als allgemeiner die "Form von Kapital" an (52), (denn ihr Charakter als „einfache Ware" oder Warenkapital ist im Zirkulationsprozeß als

48 Vgl. von Flatow/Huisken, Zum Problem der Ableitung des bürgerlichen Staates, in: Probleme des Klassenkampfs Nr. 7, a.a.O., S.,96. - Gemeint ist hier die Differenz von historischer und logischer Abfolge der Kategorien, die erst vollständig entwickelt in der Totalität der bürgerlichen Gesellschaft erscheinen. Vgl. Grundrisse, S., 28 und 163.

49 Vgl. die initiierende Funktion der Zirkulationssphäre im 16./17. Jh. in England bei der Verwandlung der Agrikultur von einer Subsistenzwirtschaft zur Produktion für den wachsenden inneren Markt: ,,So war der Zirkulation hier vorausgesetzt eine Produktion, die nur als Uberschuß Tauschwerte schuf; aber sie ging zurück in eine Produktion, die nur noch mit Beziehung auf die Zirkulation stattfand, in Tauschwerte als ihren ausschließlichen Inhalt setzende Produktion". Grundrisse, S., 168

50 ibid., S. 166

51 „Bei der kapitalistischen Produktion handelt es sich . . darum, für das der Produktion vorgeschoßene Kapital denselben Mehrwert oder Profit herauszuziehen, wie jedes andere Kapital von derselben Größe... wenigstens als Minimum, die Waren zu Preisen zu verkaufen, die den Durchschnittsprofit liefern, d.h. zu Produktionspreisen". MEW 25, S. 205

52 MEW 25, S. 882. Marx begründet dort, die Subsumtion unter kap. Verteilungsverhältnisse sei insofern z.T. richtig, ,als er es nicht seiner Arbeit verdankt, sondern dem Besitz der Produktionsmittel - welche hier allgemein die Form von Kapital angenommen haben -, daß er imstande ist, sich seine eigene Mehrarbeit anzueignen. Und ferner soweit er sein Produkt als Ware produziert, also von dem Preis desselben abhängt ..., hängt'die Masse der Mehrarbeit, die er verwerten kann, nicht von ihrer eigenen Größe, sondern von der allgemeinen Profitrate $a b^{\prime \prime}$. 
Wirkungsebene des Wertgesetzes ausgelöscht). Sie unterliegen daher der Verwandlung ihrer Werte in Produktionspreise, die Resultat ist der Verteilung des gesellschaftlichen Profits unter die Einzelkapitale durch die allgemeine Profitrate - nicht nach Maßgabe der je einzeln produzierten Mehrwertmasse sondern dem Wertanteil des Einzelkapitals am Gesamtkapital (53).

Mit dieser logischen Bestimmung ist die Voraussetzung entwickelt, von der aus die Abhängigkeit der Bereiche einfacher Warenproduktion von den vor- und nachgelagerten Sektoren kapitalistischer und zum großen Teil überdies hochmonopolisierter Produktion auf der real-analytischen Ebene als Werttransfer innerhalb der Zirkulationssphäre in seinen besonderen Ursachen und Ausprägungen erfaßt werden kann.

Obgleich der bäuerliche Warenproduzent seine Produkte allein als Tauschwerte produziert und wie der Kapitalist von der Realisierung der Warenwerte in der Zirkulation abhängt, unterscheidet ihn von diesem, daß der Zweck seiner Produktion zunächst die eigene Konsumtion ist, d.h. er gewährleistet seine eigene Reproduktion und die seiner Familie über den Verkauf selbst erzeugter Waren. Das Produkt seiner Mehrarbeit darüberhinaus beruht auf eigener Arbeit und nicht auf dem qualitativ ungleichen Tausch von Geld gegen Arbeitsvermögen, das als ,der erkaufte Gebrauchswert unmittelbar Gebrauchswert für den Tauschwert ist, d.h. wertsetzender Gebrauchswert" (54). Das von ihm eingesetzte Geld ist seiner Funktion nacb kein Kapital, insofern es kein besonderes Verhältnis zur Arbeit als Nicht-Kapital herstellt, denn ,die Bedingung der Verwandlung von Geld in Kapital ist, daß der Eigner des Geldes Geld gegen ăas fremde Arbeitsvermögen als Ware umtauschen kann“ (55). Es handelt sich also um formale Analogie, die bäuerliche nicht-kapitalistische Produktionsweise Distributionsverhältnissen zu subsumieren, die die "fetischartigste Form" der Verhältnisse kapitalistischer Produktion darstellen: "Auf Basis der kapitalistischen Produktionsweise wird es so sehr selbstverständlich, den Wert, worin sich die neu zugesetzte Arbeit darstellt, in die Revenueformen von Arbeitslohn, Profit und Grundrente zu zerfällen, daß diese Methode ... auch da angewandt wird, wo von vornherein die Existenzbedingungen jener Revenueformen fehlen" (56). Wenn daher im folgenden die Kategorien Kapital, Profit etc. verwandt werden, sind sie als formale termini technici zu verstehen; so meint der Begriff des ,variablen Kapitals" nur die unbedingt notwendigen Reproduktionskosten der Bauernfamilie (analog zum Arbeitslohn) und „Profit" das Geldäquivalent für die Arbeitszeit, die über diejenige hinaus aufgewandt wurde, die zur einfachen Reproduktion des Betriebes und der Arbeitskraft nötig gewesen wäre. 


\subsection{Integration in den kapitalistiscben Markt}

Ob der Landwirt nun nur einen Preis für seinen Arbeitslohn erzielt oder dieser ihn auch zur erweiterten Reproduktion befähigt, hängt von den Reproduktionsbedingungen $\mathrm{ab}$, die ihm die kapitalistische Warenzirkulation über den Markt aufherrscht.

Seine Abhängigkeit vom Markt verschärft sich mit der zunehmenden Ausgliederung und Verselbständigung von a) industriell oder handwerksmäßig zu betreibender Verarbeitungsfunktionen, b) von Erzeugungsfunktionen, wenn Massenproduktion in gewerblichen Betrieben profitabel möglich ist, und c) Absatzfunktionen, die ursprünglich eine organische Einheit des bäuerlichen Arbeitsprozesses bildeten und führt $\mathrm{zu}$ weitgehender Spezialisierung auf bestimmte agrarische Produkte. Damit entledigt er sich der Möglichkeit, im Krisenfall auf Eigenproduktion seiner Hilfsmittel (Saatgut, Getreide etc.) auszuweichen oder zur Subsistenzwirtschaft zurïckzukehren.

Die Frage nach den treibenden Gesetzmäßigkeiten dieser Umverteilung der gesellschaftlichen Teilarbeiten mit dem Hinweis auf ihre - auf der konkretempirischen Ebene konstatierten - Eingliederung in die vom Kapital beberrschten Sektoren zu beantworten, ist richtig, hat als Erklärung allerdings nur Plausibilitätscharakter. Vielmehr muß methodisch exakt Rekurs genommen werden auf die abstrakten Bestimmungen des Selbstverwertungszwangs des Kapitals, der unter dem Druck der Konkurrenz zur Mehrwert- bzw. Profitmaximierung auf der empirischen Ebene verschiedene Erscheinungsformen annehmen kann: Zum einen versuchen z.B. Konservenfabriken durch Direktabnahme und Lieferverträge mit den bäuerlichen Produzenten (zum Zweck der Standardisierung von Qualität, Größe, Menge etc.) ihre Kostpreise gegenüber Konkurrenten der gleichen Branche zu senken (57), zum andern kann mit Hilfe öffentlich- oder privatrechtlicher Marktorganisationen (58) den Kapitalverwertungsschwierigkeiten mebrerer Branchen der nachgelagerten Sektoren z.B. begegnet werden. Und zum dritten bildet die agrarische Produktion

57. Zur Illustration vgl. Karl Kremser, Ausbeutung der BRD-Bauern durch die Monopole der Ernährungswirtschaft. In: DWI-Berichte, Heft 11, Berlin 1971, S. 37: Der SchweisfurthKonzern, Fleischwarenproduzent mit $12 \%$ Marktanteil in der BRD, läßt die Schweine z.T. in eigenen Mastbetrieben erzeugen, z.T. erfolgt die Lieferung auf Vertragsbasis über eine bäuerliche Viehzentrale oder direkt von vertraglich gebundenen Produzenten, wobei der Konzern diese bei der Produktion und dem Einkauf der Produktionsmittel berät. Das bedeutet u.a. eine Deprivation der Bauern von ihrer Leitungs-, bzw. Planungsfunktion.

58 Eine öffentlich-rechtliche Organisation ist die "Centrale Marketinggesellschaft" (CMA), der die Durchführung der Aufgaben des Absatzfond obliegt und aus diesem finanziert wird: ,(1) Der Absatzfond hat den Absatz und Verwertung von Erzeugnissen der deutschen Land-, Forst- und Ernährungswirtschaft durch Erschließung und Pflege von Märkten im In- und Ausland mit modernen Mitteln zentral zu fördern". Gesetz über die Errichtung eines zentralen Fonds zur Absatzförderung der deutschen Land-, Forst- und Ernährungswirtschaft (Absatzfondgesetz) vom 26.6.1969. Veröffentlicht im Bundesgesetzblatt, Jg. 1969, Teil I, S. 635 ff. 
partiell (Geflügel, Schweinemast) eine Anlagemöglichkeit für überschüssiges Kapital, das in dieser Sphäre eine höhere Profitrate verspricht als in seiner ursprünglichen.

Auf der Seite der agrarischen Produzenten sind verschiedene Erscheinungsweisen der Abhängigkeit von den industriellen Markt,,partnern" auseinanderzuhalten:

Der Zwang zum Kauf selbst nicht mehr günstig zu produzierender Waren und der Verkauf auf einem nicht überschaubaren Markt unterwirft die bäuerlichen Produzenten zunächst der Regelung der gesellschaftlichen Teilung der Arbeit durch das Wertgesetz, das als ,stumme, im Barometerwechsel der Marktpreise wahrnehmbare, die regellose Willkür der Warenproduzenten überwältigende Naturnotwendigkeit“ (59) wirkt. Empirisch ist zu konstatieren: „Die Marktstellung der landwirtschaftlichen Erzeuger ist dadurch gekennzeichnet, daß einer großen Zahl von zum Teil stark spezialisierten Produzenten relativ wenige marktbedeutende Abnehmer im Ernährungsgewerbe gegenüberstehen" (60); dasgleiche gilt für das Verhältnis zum Ptoduktionsmittelsektor (61). Die Landwirtschaft als Realtypus vollständiger Konkurrenz (62) ist der Preisbildung durch die Angebot-NachfrageRelation am Markt gänzlich unterworfen (63): „Die Landwirte verhalten sich daher als Mengenanpasser, für die der Preis ein Datum ist" (64).

Auf einer zweiten Ebene ist zu untersuchen, in welchem Ausmaß und in welcher Weise sich die warenproduzierenden Marktpartner dem Wirken des Wertgesetzes entziehen und aufgrund ihrer Monopolstellung sich Surplusprofite durch nicht-äquivalenten Tausch aus der Wertmasse der agrarischen Rohstoffproduzenten aneignen.

Eine direkte Subsumtion unter hochkonzentrierte Kapitale erfolgt durch Formen der Verbundswirtschaft oder Vertikalen Integration, bei der durch Lieferkontrakte die Preisbildung am Markt in einem bestimmten Spielraum ausgeschaltet werden kann (65).

59

60

61

62

MEW 23, S. 377

Agrarbericht 73, 7/146, S. 50, Punkt 102

Arthur Hanau, Die Stellung der Landwirtschaft in der Sozialen Marktwirtschaft. In: Gerhardt/Kuhlmann (Hg.), Agrarwirtschaft... a.a.O., S.91: Die Marktform der Konkurrenz gelte in dieser Vollkommenheit nur für das landwirtschaftliche Angebot.

Josua Werner, Wettbewerb und Landwirtschaft. lbid., S. 100

3 Entsprechend der EWG-Agrarmarktordnung verstärkt der Staat allerdings die Nachfrage, insofern die Interventionsstellen Produktenquanta bei Preisfall unter den Interventionspreis (unter bestimmten Einschränkungen) aufkaufen.

4 Karin Müller-Heine, Agrarpolitische Ziele und ihre Einordnung in den gesamtwirtschaftlichen Zielkomplex. Göttingen 1972, S. 13

Ihre Verbreitung wird häufig überschätzt. L.t. E. Rechtziegler in: Westdt. Landw. . . a.a.0:, S. 87 waren Mitte der 60er Jahre $9 \%$ der landwirtschaftlichen Marktproduktion durch die Vertikale Integration erfaß̄t. Unter Einbezug der Vertikalen Integration auf gesetzlicher Grundlage bei der Milchproduktion waren es $36 \%$, die aber grundsätzlich anderen Bedingungen unterlag und daher in diesem Zusammenhang nicht betrachtet werden sollte. Außerdem wurde sie Anfang der 70er Jahre aufgehoben, was allerdings nichts daran ändert, daß der überwiegende Teil der Milchproduzenten weiterhin seine Milch an die Molkerei(genossenschaft) der jeweiligen Gegend abliefern muß. 
Erzielt der bäuerliche Produzent seine Reproduktionskosten, d.h. sein Einkommen fast ausschließlich über den Verkauf seiner Produkte (Subventionszahlungen wirken modifizierend, aber nicht grundlegend verändernd), muß eine Analyse der ökonomischen Situation der Bauern in der BRD/EWG ansetzen an der Realisierung der Warenwerte in der Zirkulationssphäre; hier liegt der analytische Angelpunkt der Disparität von agrarischen (nicht-kapitalistischem) und industriellem Sektor.

(Die restliche Hälfte dieses Artikels wird aus technischen Gründen erst in Prokla 23 erscheinen können)

hier die Gliederung der noch ausstehenden Abschnitte:

2.3.1. Preisbildung agrarischer Produkte; 2.3.2. Integration in den EWG-Agrarmarkt; 2.4. Die Inter- und intrasektorale Einkommensdisparität; 2.5. Soziale Differenzierungsprojekte im Agrarsektor - Ansätze zu einer Klassenanalyse der Bauern. / 3. Probleme der Durchsetzung bäuerlicher Interessen in einer kapitalistischen Gesellschaft. / 4. Grundzüge der Interessenartikulation durch den Deutschen Bauernverband; 4.1. Neuorientierung der Agrarpolitik Ende der 60 er Jahre; 4.2. Grundzüge der Argumentation des $D B V ; 4.3$. Forderung des $D B V$ an die staatliche Agrarpolitik; 4.4 Strukturierung, ,agrarischer" Interessen durch den DBV. / 5. Vorläufige Einschätzung der DBV-Politik

Die „Kritische Justiz" ist ein Wagnis, weil sie die in Deutsctland übliche Linie der Fachzeitschriften verläßt, auf die Fassade des Einerseits - Andererseits verzichtet und es sich offen zur Aufgabe macht, das Beharrungsvermögen und obrigkeitliche und standesmäßige Denkweise, die unsere Justiz beherrschen, durch Kritik und Polemik abzubauen ...

\section{Richard Schmid}

in "Gewerkschaftliche Monatshefte"

KAITISCHE JUSTIZ erscheint vierteljährlich, Abonnement: Jährlich 26,- DM zuzüglich Zustellgebühr, für Studenten 20,-DM, Einzelheft 7,50 DM. Bestellung an: Bund Verlag GmbH, Vertrieb "Kritische Justiz", 5 Köln, Fostfach 210140.

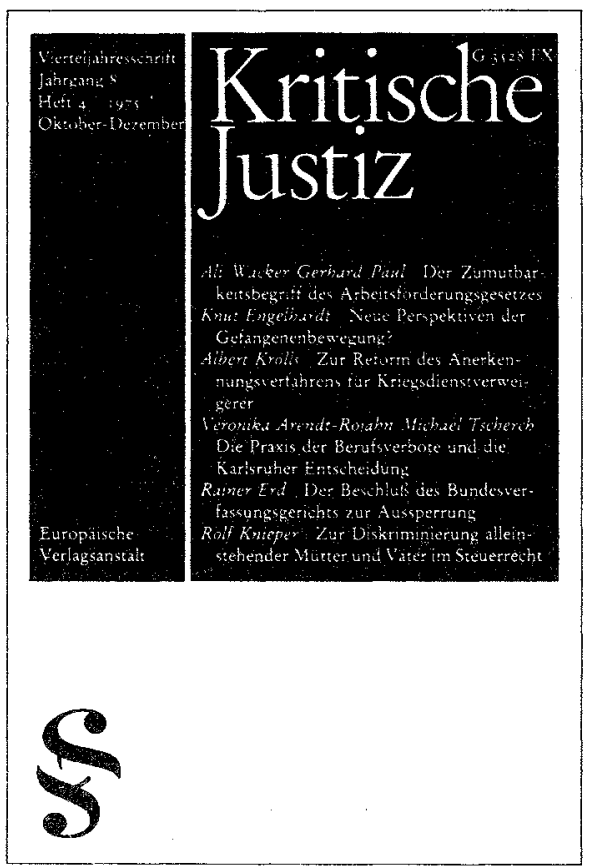

